\title{
Pattern of glomerular disease in a tertiary care hospital in Nepal: A Shree Birendra Hospital experience
}

A. Sharma ${ }^{1}$, R.K. Deo ${ }^{2}$, R. R. Shahi ${ }^{3}$

A. Sharma ${ }^{1}$, Assistant professor (NAMS), Consultant Physician, Nephrologist, Department of Medicine

R.K. Deo ${ }^{2}$,Consultant Physician, R. R. Shahi ${ }^{3}$,Consultant Radiologist Shree Birendra Hospital, Chhauni, Kathmandu, Nepal

\begin{abstract}
The incidence of renal diseases varies greatly in various parts of the world. Percutaneous renal biopsy is an important procedure for many patients with renal disease. This hospital based retrospective study evaluated the pattern of various glomerular diseases in Shree Birendra hospital.

Hospital records were searched for all the patients undergoing renal biopsy for all indication and patient profile and histopathological examination record were obtained. All the patients undergoing renal biopsy for any cause from January 2007 to December 2010 were included in the study.

A total of 46 patients underwent renal biopsy in the study period. The number of patients in the various age groups were $-<16$ years -5 patients, $16-50$ years -34 patients and $>50$ years -7 patients. The number of male and female patients was 34 and 12 respectively. Major indication for undergoing renal biopsy in this study was Nephrotic Syndrome ( $n=29,63 \%)$, followed by RPGN ( $n=8,17 \%)$, Persistent proteinuria $(n=6,13 \%)$ and Nephritic syndrome $(n=3,7 \%)$. The most common histopathological diagnosis in the study was Membranous nephropathy $(\mathrm{n}=10,22 \%)$, followed by Focal Segmental Glomerulosclerosis $(n=9,20 \%)$, Membranoproliferative glomerulonephritis $(n=9,20 \%)$ and Minimal change disease $(n=6$, $13 \%)$.

USG guided renal biopsy is safe in adults and is a very useful tool for the diagnosis of renal disease. The pattern of glomerular disease varies in different settings. There is a need for further evaluation of glomerular disease with larger sample size to determine the pattern of glomerular disease in Nepal.
\end{abstract}

Keywords: Glomerular disease, renal biopsy, Nepal.

\section{Introduction}

Renal diseases are increasing day by day. The incidence of renal diseases varies greatly in various parts of the world. Epidemiologic studies on renal biopsies are the best way to monitor glomerular

Correspondence: A. Sharma

E-mail: disease trends. Histological examinations of the biopsies from kidneys are the gold standard for diagnosis of renal diseases.

Percutaneous renal biopsy is an important procedure for many patients with renal disease. It was first reported in 1934 by Ball and became a routine procedure later. ${ }^{1}$ The kidney was originally localized by correlating plain radiographs with 
A. Sharma et al, Pattern of glomerular disease in a tertiary care hospital in Nepal..

anatomical landmarks. ${ }^{2}$ Various methods have been used to localize the site of kidney for biopsy which includes plain radiographs, image intensification fluoroscopy with pyelography, ultrasound and computed tomography. The use of real time ultrasonography together with the invention of automated core biopsy gun has made the task of performing renal biopsies safer, easier and faster. ${ }^{3}$ In 1951, Inverson and Brun reported the first large series of needle biopsies of kidney, ${ }^{4}$ subsequently hundreds of papers have been published reporting on the technique, ease and safety of precautious renal biopsies in different age groups.

This study evaluates the histopathological patterns of biopsies obtained from kidney for various indications in Shree Birendra Army hospital, Nepal from 2007 to 2010.

\section{Materials and Methods}

Hospital records were searched for all the patients undergoing renal biopsy for all indication. Patient profile and histopathological examination record of all the patients were obtained. All the patients undergoing renal biopsy for any cause from January 2007 to December 2010 were included in the study. This was a retrospective hospital records based study. The objectives were to analyse indication of renal biopsy, adequacy of samples obtained and pattern of glomerular disease in the given period of time.

Biopsy samples were obtained using automated Gun under ultrasound guidance. 2-3 punctures were done to obtain adequate sample, which was confirmed by gross examination. Biopsy samples were considered satisfactory for diagnosis if they contained seven or more glomeruli. All the samples were subjected to light microscopic examination. Immunoflorescent examination was not done.

Patients were admitted and observed for 24 hours after biopsy. The complication looked for was hematuria and pain at the biopsy site. The complications observed in this study were mild, none requiring any major intervention.

\section{Results}

A total of 46 patients underwent renal biopsy in the study period. The number of patients in the various age groups were $-<16$ years -5 patients, $16-50$ years -34 patients and $>50$ years -7 patients (Table 1).

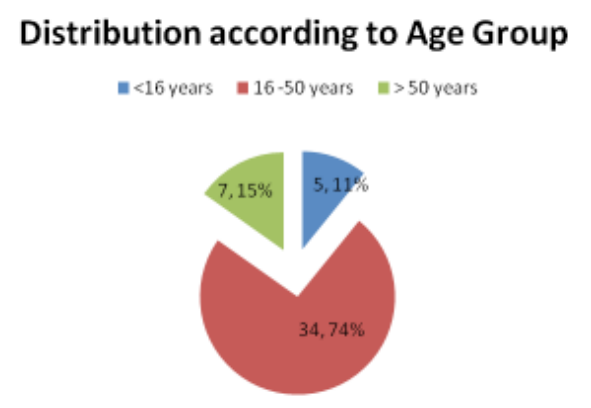

Table 1. Distribution of patients according to Age Group

The number of male and female patients was 34 and 12 respectively (Table 2). 


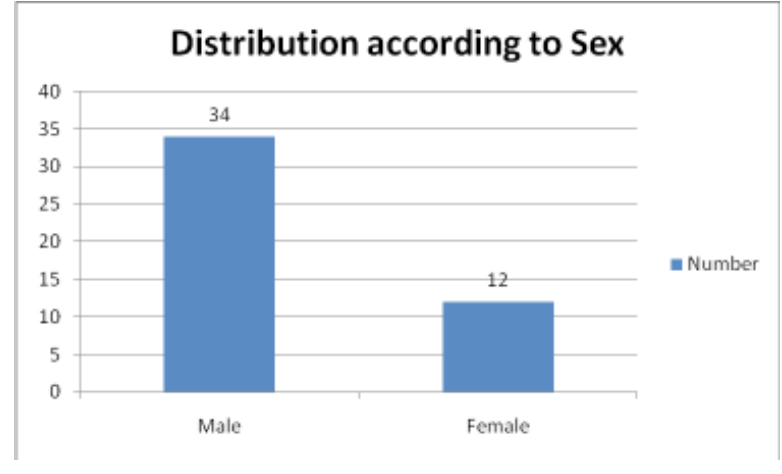

Table: 2. Distribution of patients according to Sex

Major indication for undergoing renal biopsy in this study was Nephrotic Syndrome ( $n=29,63 \%)$, followed by RPGN ( $n=8,17 \%)$, Persistent proteinuria $(\mathrm{n}=6,13 \%)$ and Nephritic syndrome $(\mathrm{n}=3,7 \%)$ (Table 3$)$

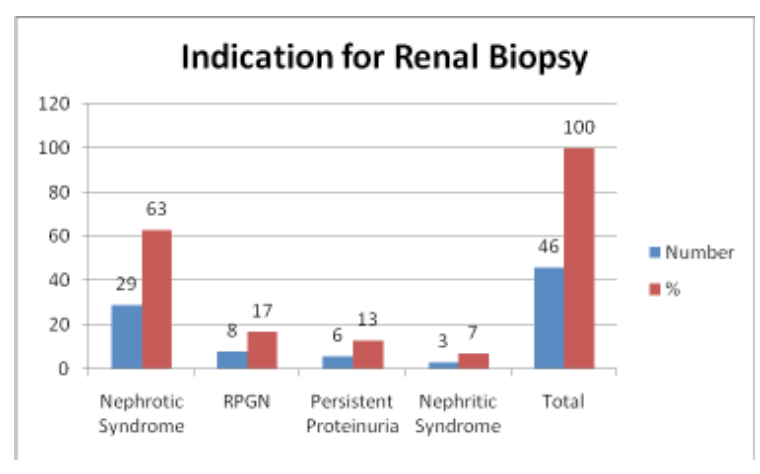

Table: 3. Indication for renal biopsy

The most common histopathological diagnosis in the study was Membranous glomerulonephritis $(\mathrm{n}=10,22 \%)$, followed by Focal Segmental Glomerulosclerosis $\quad(n=9, \quad 20 \%)$, Membranoproliferative glomerulonephritis $(\mathrm{n}=9$, $20 \%)$ and Minimal change disease $(n=6,13 \%)$. Other diagnosis was as shown in the table.
Table: 4

$\begin{array}{lll}\text { Diagnosis } & \text { No. of cases } & \text { \% } \\ \text { MN } & 10 & 22 \\ \text { FSGS } & 9 & 20 \\ \text { MPGN } & 9 & 20 \\ \text { MCD } & 6 & 13 \\ \text { PIGN } & 5 & 11 \\ \text { Focal Interstitial Nephritis } & 3 & 6.5 \\ \text { Necrotizing GN } & 1 & 2.2 \\ \text { CGN } & 1 & 2.2 \\ \text { CIN } & 1 & 2.2 \\ \text { Hypertensive Nephropathy } 1 & 2.2 \\ \text { Total } & \mathbf{4 6} & \mathbf{1 0 0}\end{array}$

Table: 4. Histolopathological Diagnosis

Abbreviations : MN,Membranous nephropathy; FSGS, Focal Segmental Glomerulosclerosis; MPGN, Membranoproliferative glomerulonephritis; MCD,Minimal change disease; PIGN, Post infectious glomerulonephritis; CGN, Chronic glomerulonephritis; CIN, Chronic interstitial nephritis.

\section{Discussion}

The most common indication for undergoing renal biopsy in our study was Nephrotic Syndrome which is higher as compared to other studies. $5,6,7$

The most common histopathological diagnosis in the study was Membranous nephropathy (MN), followed by Focal Segmental Glomerulosclerosis (FSGS), Membranoproliferative glomerulonephritis (MPGN) and Minimal change disease (MCD). The incidences of various glomerulonephritis are also varied as compared to other studies. In a study done in Spain, the most common diagnosis was MCD followed by FSGS. ${ }^{8}$ 
A. Sharma et al, Pattern of glomerular disease in a tertiary care hospital in Nepal..

The study in France had IgA nephropathy and MPGN as leading cause of glomerular disease. ${ }^{9}$ Study done in Netherlands showed MCD and IgA nephropathy as common glomerular disease. ${ }^{10}$ MCD was most common glomerular disease in Czech study. ${ }^{11}$ Study in Romania showed MPGN as leading cause of glomerular disease. ${ }^{12}$ The incidence of MN and FSGS were higher in our study as compared to Australian study. ${ }^{13}$ A Brazilian study showed higher incidence of FSGS as compared to MN. ${ }^{14}$ Danish registry showed the highest incidence of MN followed by MCD and FSGS. ${ }^{7}$ Study from Jordan also showed similar results as Danish. ${ }^{15}$ Other diagnosis in our study were - Post infectious glomerulonephritis, Focal interstitial nephritis, Necrotizing glomerulonephritis, Chronic glomerulonephritis, Chronic interstitial nephritis and Hypertensive nephropathy.

There were no significant side effects seen after the procedure in the study. Few patients developed pain at the site of biopsy and some developed hematuria. The complications were minor and all were managed conservatively. All patients were discharged after 24 hours observation. This finding also correlates well with other studies. ${ }^{16}$

\section{Conclusion}

We conclude that USG guided renal biopsy is safe in adults and is a very useful tool for the diagnosis of renal disease. The pattern of glomerular disease varies in different settings. There is a need for further evaluation of glomerular disease with larger sample size to determine the pattern of glomerular disease in Nepal.

\section{References}

1. D.P. Ball, Needle (aspiration) biopsy. J Tennessee Med Assoc 1934; 27:203-6.

2. R.M. Kark, R.C. Muehrcke. Biopsy of kidney in the prone position. Lancet 1954; 266:1047-9.

3. S.S. Yesudas, N.K. Georgy, S. Manickam, Percutaneous real-time ultrasound-guided renal biopsy performed solely by nephrologists: A case series. Indian J Nephrol 2010;20:137-41.

4. P. Inversen, C. Brun. Aspiration bipsy of the kidney. Am J Med 1951; 11:324.

5. L. Gesualdo, A.M. Di Palma, L.F. Morrone et al. The Italian experience of the national registry of renal biopsies, Kidney Int 2004; 66: 890-4.

6. F.P. Schena. Survey of the Italian Registry of Renal Biopsies. Frequency of the renal diseases for 7 consecutive years. Nephrol Dial Transplant 1997;12: 418-26.

7. J. Heaf . The Danish renal biopsies register. Kidney Int 2004; 66: 895-7.

8. F. Rivera, J.M.L. Gomez, R.P. Garcia et al. Clinicopathologic correlations of renal pathology in Spain. Kidney Int 2004; 66: 898-904.

9. P. Simon, M.P. Ramee, R. Boulahrouz et al. Epidemiologic data of primary glomerular diseases in western France. Kidney Int 2004; 66: 905-8.

10. P. Van Paassen, P.J.C. Van Breda Vriesman, H. Van Rie et al. Signs and symptoms of thin basement membrane nephropathy: A prospective regional study on primary glomerular disease-The Limburg Renal Registry. Kidney Int 2004; 66: 909-13. 
11. I. Rychlik, E. Jancova, V. Tesar et al. The Czech registry of renal biopsies. Occurrence of renal diseases in the years 1994-2000. Nephrol Dial Transplant 2004;19: 3040-9.

12. A. Covic, A. Schiller, C. Volovat et al. Epidemiology of renal disease in Romania: a 10 year review of two regional renal biopsy databases. Nephrol Dial Transplant 2006; 21: 419-24.

13. E.M. Briganti, J. Dowling, M. Finlay et al. The incidence of biopsy-proven glomerulonephritis in Australia. Nephrol Dial Transplant 2001; 16: 1364-7.
14. M.G. Polito, L.A. Ribeiro de Moura, G.M. Kirsztajn. An overview on frequency of renal biopsy diagnosis in Brazil: clinical and pathological patterns based on 9617 native kidney biopsies. Nephrol Dial Transplant 2010; 25:490-6.

15. M. Ghnaimat, N. Akash, M. El-Lozi. Kidney Biopsy in Jordan: Complications and Histopathological Findings. Saudi J Kidney Dis Transpl 1999;10:152-6.

16. M. Rahbar. Kidney biopsy in west of Iran: Complications and histopathological findings. Indian J Nephrol 2009;19:68-70. 